\title{
Research on a Method and Case of Users' Participation in Product Innovative Design
}

\author{
Ertian Hua ${ }^{1}$, Jiaji Zhong ${ }^{1, *}$, Lei $\mathrm{Hu}^{2}$, Qizong Sun ${ }^{1}$ and Chuan Chen ${ }^{1}$ \\ ${ }^{1}$ Zhejiang University of Technology, Hangzhou, Zhejiang, China, 310032 \\ ${ }^{2}$ Zhejiang Gongshang University, Hangzhou, Zhejiang, China, 310018 \\ ${ }^{*}$ Corresponding author
}

\begin{abstract}
According to the features of abundance, diversification and short-period in consumer product innovative design, an efficient method of users' participation in product innovative design was put forward: Percolating the professional users by analyzing online comments to products; Requiring the experiences and recommendations from professional users by online interaction; Promoting the efficiency and competitiveness of product innovative design by the online collaborative design between enterprise $R \& D$ staffs and professional users. Finally, the existing cases are analyzed.
\end{abstract}

Keywords-product innovation; design methodology; user screening; online interaction

\section{INTRODUCTION}

Chinese manufacturing industry is on a key period of structural adjustment and change in the development pattern[1]. It is being a crucial part of Chinese economy which is transformation to an innovation driven pattern and upgrading[2]. With the deep integration of industrialization and informatization, it is more and more obvious that consumer product innovation present abundance, diversification and short-period features. In Anderson and Marsh's idea, the industrial revolution of customized mass production is on the rise[3][4]. Traditionally informational technology enterprises get more opportunities to take part in the innovation of manufacturing industry, which promotes the transformation from traditional manufacturing enterprises to cross-order integration enterprises[5].

The vast majority of traditional product innovative design are achieved by professional designers who're in the enterprises. According to the collection of market information , enterprises put forward constraints on product innovative design, by which designers accomplished the design .Risks exit in exploitation of new product on account of the limitation of product innovative information that collected by enterprises. Information asymmetric problems in the product innovative activities are constantly solved with the improving of informational technology. On the one hand, with the integration of industrialization and information, a low-cost connection can be achieved between users and manufacturers, by which product design and manufacturing information get more transparent. On the other hand, users' participation design being on the rise, which caters the consumers' consumptive concept of active participation, entertainment sharing, high quality and inexpensive. Which transforms users into designers and disseminators from passive receivers[6].
Thus it is a high concern in academia and industry that how to rapidly and effectively extract excellent product innovative ideas from the huge numbers of users' feedback. It was emphasized by Wisdom Manufacturing mode proposed by Xifan Yao, etc, that collective wisdom and users' participation in common innovation[6][7]. Weili Huang, etc, hold that the fundamental starting point of modern design is users' requirements analyzing[8]. The above studies affirmed the importance of users' participation, but the professional levels of the users has not yet be distinguished. An efficient innovative design method was proposed by this paper, efficiency of which was reflected in the selection of users with professional background, and encourage users deeply participate in the product innovative design. Professional users combine the professionalism and marketability, so their expression is more precise and effective, and their solutions are more feasible. Analyses, namely products' characteristics and consumers' attitudes, tendency and emotion in the product evaluation were respectively accomplished[9]. Based on the automobile evaluation information, analysis on the meanings of the consumers' evaluation was accomplished[10]. Combined with LTP technology, a knowledge base was built to determine the emotional tendencies of polar words[11]. The interaction between design and manufacturing interfaces of visual enterprise was studied in Literature [12]. Concrete method of this paper is that analyzing and percolating the professional users by analyzing online comments; Obtaining their experiences and recommendations by online interacting between enterprise and professional users; And do online collaborative design on the enterprises' online design platform. This paper aims to provide a kind of innovative design idea which can reflect the users' intentions more effectively and improve the success rate, pertinence and market competitiveness of the product innovative design on the background of difficult customization.

\section{Percolating Professional Users}

\section{A. Creating Collections Of The Key Attributes}

Users' effective demands can be picked out by using the comments data. As shown in figure 1, first is the key attributes of products should be determined. The key attributes of products usually consisted of two parts: products' existing attributes and expected attributes. The products' existing attributes were determined by enterprise $\mathrm{R} \& \mathrm{D}$ staffs, as in

$$
U_{o}=\left\{v_{o 1}, v_{o 2}, \ldots, v_{o n}\right\}
$$


Where $\mathrm{n}$ denotes the number of products' existing attributes and von denotes the nth attribute value.

Products' expected attributes U'0 should be obtained by online comments mining.

The collection of products' expected attributes as in

$$
U_{o}^{\prime}=\left\{v_{o 1}^{\prime}, v_{o 2}^{\prime}, \ldots, v_{o n}^{\prime}\right\}
$$

Where $\mathrm{n}$ denotes the number of products' existing attributes and v'on denotes the nth attribute value.

Combining the products' existing attributes U0 and expected attributes U'0, and optimized and screened by design teams of enterprises. The collections of products key attributes V0 which is truly concerned by consumers were obtained, as in

$$
V_{0}=\left\{v_{1}, v_{2}, \ldots, v_{n}\right\}
$$

Where "n” denotes the number of key attributes.

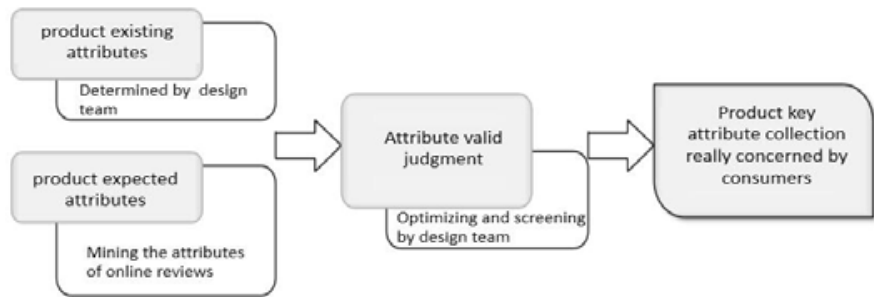

FIGURE I. PROCESS OF BUILDING PRODUCTS’ KEY ATTRIBUTES COLLECTION

In the actual comments, knowledge attributes of products' design exist a vast number of qualitative data, which need to be quantified. If the number $\mathrm{j}$ attribute $v_{i j}$ is a qualitative data, $\mathrm{k}$ types of qualitative data and $v_{i j}$ is the number $p(p \leq k)$ type of qualitative data, then these qualitative attributes were quantified as in

$$
v_{i j}^{\prime}=\frac{p}{k}, v_{i j}^{\prime} \in[0,1]
$$

As different data have different dimensions, typically, data need standardized and compressed to the standard range $[0,1]$, namely transforming into standard deviation:

$$
v_{i k}^{\prime}=\frac{\left|v_{i k}-\overline{v_{k}}\right|}{s_{k}} \quad(i=1,2,3, \ldots n \quad k=1,2,3, \ldots, m) ;
$$

where $\overline{v_{k}}=\frac{1}{n} \sum_{i=1}^{n} v_{i k}, s_{k}=\sqrt{\frac{1}{n} \sum_{i=1}^{n}\left(v_{i k}-\overline{v_{k}}\right)^{2}}$

In the actual process, users' comments are mostly in the form of natural language with multiple clauses, which is the computer difficult to distinguish and handle. For Chinese words without separators between the words, thus, the participles processing is performed before the feature words are extracted; For the same comments on products' attributes expressed in different terms, template language was used to deal with synonym extensions for a word; For example, HIT(Harbin Institute of Technology) CiLin (extended version) is divided into large, medium, small three classes[13], in which for each word in the corpus, the synonym template for the synonym of the composition can be obtained by searching the synonym phrases. If the two templates are similar, both of them are merged to reduce redundancy and improve retrieval speed. For the candidate library which has been merged, its reliability can be judged by comparing the number of occurrences of the templates, and a higher frequency of occurrence template determines higher reliability; the templates containing the search term are retrieved in the template library, the sequence numbers in the templates are retrieved, and the matched search is performed using the initial position of the same subsequence with Data Processing System in the position recorded template; Finally, the found template nodes and position can be used to locate the attribute words in the comments. Assuming that the node value in the template is $\omega$, the word corresponding to position $+\omega$ in the comment word is the attribute word.

\section{B. Judgment On Comments Information Validity}

The valuable content for the product innovation has to be dug out in the massive and obscure comments data. Firstly, the target data have to be confirmed based on the products' key attributes that has been found, according to which the real valuable data information has to be mined, as shown in fig2. In this paper, we proposed a method to filter the data of the comments by sorting the data, find out the key attributes of a product that are common to a set of data objects from comments data, and divided them into several categories according to the classification model of the product's attributes, some of which were defined by the product designers as professional-class. Before the classification analysis, the key attributes are clustered to classify the data that similarities or differences, so that the similarity between the similar types of data became as large as possible, and the similarity between different types of data became as small as possible. In addition, the key attributes of the products may existed the established relationship, that is, the emergence of a key attribute may accompany the emergence of another product attributes, which needs to integrate with the classification method. The data items in the comments are mapped to a given category by the classification model. The Euclidean Distance Method in the direct distance methods was used to compare the similarity roi between each category and professional category:

$$
r_{o i}=d\left(U_{0}, U_{i}\right)=\sqrt{\sum_{k=3}^{m}\left(v_{o n}^{\prime}-v_{i n}^{\prime}\right)^{2}} \quad n=3,4,5, \ldots \ldots .
$$

Where v'on, v'in are the normalized attribute values.

A threshold $\delta$ was determine based on existing design experiences and consumer groups' preferences, according 
which the obtained comments can be judged effectively. The validity of NO i online comment was set to be $t_{i}$, as in

$$
t_{i}= \begin{cases}1 & r_{o i} \leq \delta \\ 0 & r_{o i}>\delta\end{cases}
$$

Where $t_{i}=1$ means that the comments were valid; otherwise, the comments were invalid (discarded).

Finally, a set of valid comments was obtained as $W=\left\{w_{1}, w_{2}, \ldots, w_{m}\right\}$, where $m$ denotes the number of valid comments finalized.

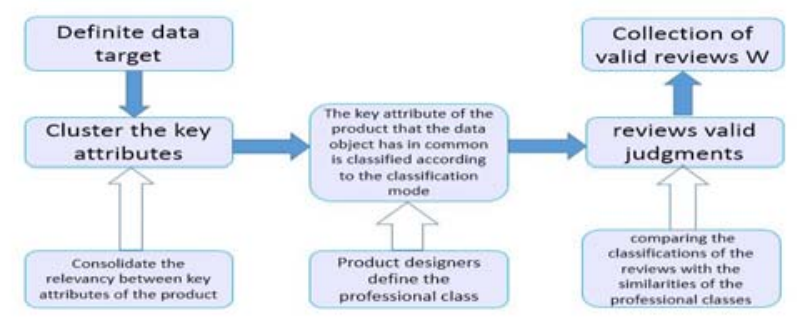

FIGURE II. METHODS OF CLASSIFYING AND ANALYZING COMMENTS DATA FOR VALIDITY

\section{Professional Users Selection}

According to the results of 1.2, each user whose comments are validated with effectiveness was found. The product's attributes of user i comments was set to be $\mathrm{Vi}=\{\mathrm{vi} 1, \mathrm{vi} 2, \ldots, \mathrm{vin}\}$, if a certain attribute satisfies vin $\in \mathrm{V} 0$, it accords with the survey result, denoted as $\lambda_{n}=1$, otherwise $\lambda_{n}=0$. With threshold set as $\mathrm{m}$, the user whose comments attributes meeting criteria accumulate to $\mathrm{m}$, was valid to be a professional user, denoting $T_{i}=1$, otherwise $T_{i}=0$, as in

$$
\lambda_{n}=\left\{\begin{array}{ll}
1 & V_{\text {in }} \in V_{0} \\
0 & V_{\text {in }} \notin V_{0}
\end{array} T_{i}= \begin{cases}1 & \sum_{j=1}^{n} \lambda_{j} \geq m \\
0 & \sum_{j=1}^{n} \lambda_{j}<m\end{cases}\right.
$$

Three examples about a complete statement of the automatic transmission were given below: 'This vehicle is difficult to accelerate', 'It is undefined for automatic transmission to get the intentions of driver', 'Automatic transmission's shift logic is not clear, for which deep step on the acceleration fails to downshift timely'. The above three statements are typical description of the automatic transmission feature information, from which the professional level of users can be discovered. User A proposed the weakness of acceleration while the user is not clear with vehicle; user B knows that the reason for weak acceleration source of the issue is AT vague to the intentions of driver, while the user is not clear with the source of the issue; user $C$ proposed not only the shift logic, but also the source of the issue that is slowness of AT downshift. According to feedback of the above three users, it is easily discovered that three users have different professional level to the product. Among the three users, user $\mathrm{C}$ is the most professional, with whom motor corporations can establish deeper contact via his source place and domain name.

\section{FRAMEWORK OF INNOVATIVE DESIGN PLATFORM}

\section{A. Platform Basic Function And Its Expansion}

Based on the statements in first part of this paper, after discovering professional users interested by enterprises, the innovative design platform aims to establish contact between enterprise R\&D staffs and professional users, which promotes a deeper communication on design and discussion on improvement project of product innovation. Because of the small number of professional users, the platform is aimed at a tiny minority of user groups, from the perspective of which it can achieve close collaborative communication between enterprises and users, but also lower costs that enterprise design platform opens to professional users.

The basic function of the platform is to achieve the most basic network architecture for design communication. The R\&D staffs and users used the same online 3D design software to solve the design problem what is difficult to communicate by language. The platform will be extended for users to add some features, such as allowing $R \& D$ staffs and users to achieve real-time voice and text communication, and expansion must use the open network version of the software. Using VR technology, professional users connect with the virtual reality equipment of enterprise $R \& D$ staffs to enter the same virtual space and form a short-period 'virtual group' by intuitive communication and collaboration. This kind of distributed virtual reality technology breaks the limitation of time and space, realizes the exchange of ideas in real time, be able to ague the research plan in time, share the data of simulation experiment, and so on, which will greatly improve the efficiency of knowledge innovation.

In order to improve the overall design speed, groups were emphasized in the process of design communication. In term of enterprise, organized design, process manufacturing and service support staffs form the certain numbers of functional groups, and dock with individual user respectively, as shown in fig.3. Design, manufacture, service, management and user should be integrated into a coherent whole, using CIMS(computer integrated manufacturing systems) and concurrent engineering technology to coordinate the work of all personnel, in the case that all individuals, including users were used the same terminology and share information resources. The digital assembly was applied to find the interference, coordination, and increased the number of feedback in the design process, optimized and accelerate the design process. 


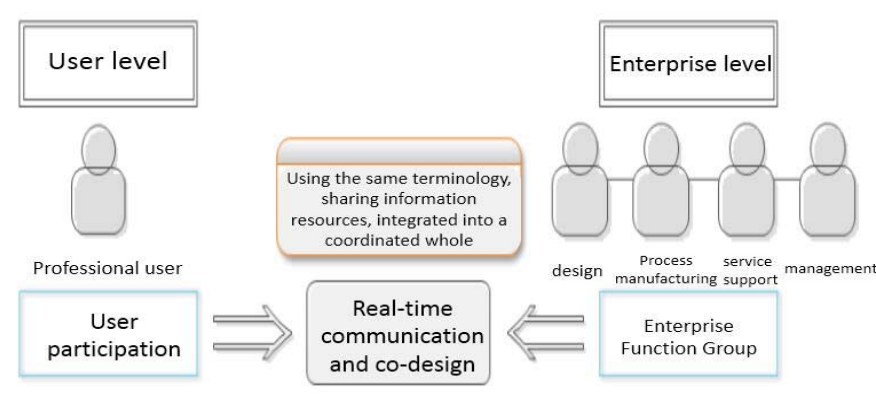

FIGURE III. DESIGN COMMUNICATION BETWEEN ENTERPRISE AND USERS

\section{B. Point-To-Point Collaborative Design For Specific Products}

\section{1) Subject design}

Point-to-point refers to the enterprise design group in the individual design with a single professional user by online communication, the user can also design with multiple designers of group. Different from the traditional collaborative design, user-based collaborative design needs to rely on online design platform, so that different regions of professional users and enterprise designers to design and communicate in-time.

Considering the obligation of the users to participate in the product innovative design, the topic of interactive design should be repeated before the online collaborative design. The users' concerns should be analyzed, but also the possible problems, products' problems and possible innovative points should be analyzed. Understand what are hope to solve through interaction, and so on. Combined with the analysis of effectiveness and feasibility which proposed by users' innovation, determined the interactive theme from a professional point of view and products sale experiences.

Particularly, the mining of potential users' involvement in the early stages should be taken seriously. Some potential professional users had made good recommendations, but follow by a lack of focus on product's optimization and innovation, so an incentive method to attract such users is very important. For example, participation in the results of innovation can receive the corresponding points to upgrade and rewards. The user can purchased products in preferential price or required direct incentives if the user's proposal was adopted in the development of new products. Thus, users' consumptive concept of active participation, entertainment sharing, high quality and inexpensive were achieved.

\section{2) Communication and interaction}

Typically, the number of qualified professional users who meet the requirements was small, communication and interaction can be achieved by given the users a temporary lo-gin and password of internal design platform. That is, during the online collaborative design, enterprise designers and professional users achieved innovative ideas and technical communications by sharing internal design platform. Virtual reality will make this collaborative design process more intuitive and vivid. For the situations of products which had high frequency of innovation or existed more professional users, it is recommended that enterprises developing a dedicated design platform. In this process, the application of computer-aided intelligent design should be considered. For some of the conventional design rules, design standards would be determined or directly rounded by dedicate design platform. As mentioned in Literature [14], a smart decision-making system was proposed for intelligent design of motorcycles.

Further, in order to improve the efficiency of online interaction, a dedicate design platform can develop an automated question and answer software. First of all, the users' online comments for word processing, users' comments recorded in the products' key attributes, and further quantitative calculation of the user's level of specialization of the property $n$, expressed as $v_{n m}$, if the user's comments for a property have appeared multiple words, then selected the highest word to represent, as in $v_{n m}=\max V_{n 1}, V_{n 2}, V_{n 3} \ldots$. Finally got the user's specialized level array, as in

$$
A_{i}=v_{1}, v_{1 m}, v_{2}, v_{2 m}, \ldots, v_{n}, v_{n m}
$$

Where $A_{i}$ is the comments of user $i, v_{n}$ is whether attribute $\mathrm{n}$ was mentioned or not, and if the user refers to attribute $n, v_{n}=1$; if not, $v_{n}=0$.

Secondly, according to the levels of specialization of different users, the generation of targeted user feedback interactive content.

A. Use the products' key attributes mentioned in the user comments to determine the interactive Q\&A attribute module $Q_{i}$ :

$$
Q_{i}=\left\{\begin{array}{ll}
1 & v_{i} \in V_{0} \\
0 & v_{i} \notin V_{0}
\end{array} ;\right.
$$

Where $\mathrm{Qi}=1$ means that the interactive $\mathrm{Q} \& \mathrm{~A}$ will contain the number i key attribute on the product; otherwise, the interactive Q\&A was not included the content of the number $\mathrm{i}$ key attribute of the product.

B. The key attributes of the same product, their interactive content was not the same by different levels users of specialization, if $\mathrm{Qi}=1$, then by the following formula to selected interactive content with the user:

$$
Q_{i j}=v_{n m}
$$

Where $Q_{i j}$ denotes the user interaction content with degree $j$ of the product key attribute $i$.

C. Generate an online interactive Q\&A for such users, as in

$$
Q=\left\{Q_{1 j}, Q_{2 j}, \ldots, Q_{n j}\right\} .
$$


Online interaction process, and sometimes the users' needs related to a number of product features, corresponding to multiple functional design[15]. At this point, to evaluate the similarity of each functional design, similarity index calculated with existing products, the higher index means the program and the greater the similarity of existing products. This situation can be more dependent on the existing enterprise infrastructure and capacity to reduce the difficulty of subsequent design and manufacture and cost[16]. Enterprises should be based on their manufacturing capacity, time, economic benefits and other factors, to determined a similarity threshold. If a scheme similarity index is lower than the limit of the threshold, it indicates that the difference between the scheme and the existing product is too large, which will increase the difficulty of subsequent design and manufacture and affected the enterprise's benefit. In this case, the enterprise designers should communicated with the user in-depth improvement program to guide the feedback adjustment to achieve the user's needs and business status of the optimal match to form a final innovative design.

\section{3) Knowledge extraction}

Interaction process is the process of product innovative design what's very active in the process of new ideas, the background should be recorded user experience, improve the proposal and the two sides of real-time exchange of information in real-time, which in order to post-view. Including knowledge, techniques and methods that are multidisciplinary, integrated and integrated into the knowledge base and method library after combing and extracting. The acquisition, presentation and operation of various types of information and resources should also be documented, managed and analyzed with a view to continuous improvement and improved collaborative design efficiency.

\section{CASE STUDY}

In recent years, the Internet reparateur showed explosive growth. Since March 2015, Alibaba, Baidu, Tencent, Apple and other more and more Internet companies have announced their reparateur plan. But for now, the Internet enterprise car facing many problems. First of all, Internet companies in the accumulation of technology of reparateur was almost zero; Second, the traditional long-term R\&D cycle of the automotive industry was incompatible with the concept of fast-disappearing of Internet industry.

In addition to the Internet business-oriented reparateur mode, the car-led Internet car is more worth to exploring. Chery Holding Group had set up an independent Crowd sourcing brand "COWIN". The COWIN automotive use of Internet thinking, to take is integrated large data, cloud platform, the new "Crowd sourcing" mode of research and development means to intelligent interconnection as the reparateur core. At present, online users can register through their various functional modules to participate in interactive, receive the appropriate points to upgrade and get the award. If a user's proposal is accepted in the development of a new product, the user can purchase the product at a favorable price. This model stimulated the users' enthusiasm and initiative from the subjective feelings. This paper presented the following points: Obtain The collections of products key attributes which is truly concerned by consumers, as described in 1.1. The data items in the comments are mapped to a given category by the classification model. The Euclidean Distance Method was used to compare the similarity by effective criterions, what are obtained from the classification model of 1.2. Dig out potential users and to established the contact with them, provide temporary user name and password, to its partial open enterprise design platform. Enterprise design team with the users' concern, from a professional point of view and products' sale experiences, users' innovative ideas for the effectiveness and feasibility analysis to determine the theme. The both sides use the same terminology, sharing information resources, a deeper level of communication and online collaborative design, which made users and enterprises to achieved the integration of innovation and technology.

In this process, computer-aided intelligent design was essential, it was proposed by Rong Dai, etc, that a smart real-time decision-making system for intelligent design of motorcycles[14]. For a car, as a complete vehicle system is more complex than a motorbike, and a single component change can cause a series effect, in order to avoid component interference, the computer's intelligent assistance will be more comprehensive. Such as professional-level design of the body details of the digital simulation in wind-driven experiment, the follow-up will focus on the content of this study.

\section{V.CONCLUSION AND PROSPECT}

Users' participation in product innovative design is a topic of great concern to the industry and academia, with the help of the Internet platform and information technology to develop public innovative resources, to seek greater than individual groups of wisdom. In this paper, an efficient method of users' participation in product innovative design was put forward: through the establishment of products' key attributes set, the effectiveness of online comments content to determined, selected the professional users; the basic structure, basic functions and extended functions of the collaborative innovation design platform are analyzed; a simple and convenient point-to-point online collaborative design scheme is proposed for a specific product to realize low-cost collaborative product innovation design, at the same time to a certain extent to solve the traditional design model innovative capacity and innovative shortage of human resources problems, get rid of the passive product to adapt to the traditional market approach to improve products' efficiency and competitiveness in the market. Users' participation in innovative design is a subject under study, for the whole machine, a single component change may cause a series effect, in order to avoid interference, the network, intelligence and data support of design platform was crucial.

\section{ACKNOWLEDGMENT}

Project supported by the National Natural Science Foundation, China(No.71373242).

\section{REFERENCES}

[1] LU Yongxiang,On the way to green and intelligent manufacturing[J].China Mechanical Engineering, 2010, 21(04): 379-386,399(in Chinese). 
[2] ZHOU Ji.Intelligent mannfacturing-main direction of "made in China 2025”[J]. China Mechanical Engineering, 2015,26(17): 2273-2284(in Chinese).

[3] Anderson C.Makers:the new industrial revolution[M]. New York, USA: Random House,2012.

[4] Marsh P.The new industrial revolution: consumers, globalization and the end of mass production[M]. New Haven, USA: Yale University Press, 2013.

[5] WANG Xiwen.Industry 4.0:The last time the industrial revolution[M]. Bei-jing:Publishing House of Electronics Industry, 2015(in Chinese).

[6] YAO Xifan,LIAN Zhaotong,YANG Yi,et al.Wisdom manufacturing: new humans-computers-things collaborative manufacturing model[J].Computer Integrated Manufacturing Systems, 2014, 20(6):1490-1498(in Chinese).

[7] YAO Xifan,Li Bin, Dong Xiao-qian,et al.Integrated framework of wisdom manufacturing systems from semiotic perspective[J]. Computer Integrated Manufacturing Systems, 2014, 20(11):2734-2742(in Chinese).

[8] HUANG Weili,SHI Dongmin.Product innovation design oriented to user requirements[J]. Journal of Machine Design,2014(11) : 18-20(in Chinese).

[9] Liu B, Hu M, Cheng J. Opinion Observer: Analyzing and Comparing Opinions on the Web[C]// In WWW '05: Proceedings of the 14th international conference on World Wide Web. 2005:342--351.

[10] Gamon M, Aue A, Corston-Oliver S, et al. Pulse: mining customer opinions from free text[C]// In Proc of International Symposium on Intelligent Data Analysis. Springer-Verlag, 2005:121-132.

[11] Yi J, Niblack W. Sentiment Mining in WebFountain[J]. Data Engineering. icde .proceedings. international Conference on, 2005:1073-1083.

[12] Ripamonti L A, Peraboni C A. Managing the design-manufacturing interface in virtual enterprises through multi user virtual environments: a perspective approach[J]. International Journal of Computer Integrated Manufacturing, 2010, 23(8-9):758-776.

[13] HIT IR-Lab Tongylcl Cllln(Extended)[OL].HIT IR Lab. http://ir.hit.edu.cn/(in Chinese).

[14] DAI Rong,HE Yulin,YANG Xiangang,et al. Application Research on Case -based and Rule -based Reasoning Integrated in Intelligent Design of Motorcycle[J]. China Mechanical Engineering, 2008, 19(11):1363-1368(in Chinese).

[15] LIU Fei,LI Shixin,LIU Shen.The idea and application of collaborative configuration of user requirements for mass customization[J]. Journal of Mechanical Engineering,2004， 40(1):109-113(in Chinese).

[16] Sa'ed M. Salhieh, Ali K. Kamrani. Macro level product development using design for modularity[J]. Robotics and Computer-Integrated Manufacturing, 1999, 15(4):319-329. 